\title{
Identifying and controlling phase purity in 2D hybrid perovskite thin films
}

Yinghong Hu, ${ }^{1}$ Laura M. Spies, ${ }^{1}$ Diego Alonso-Álvarez, ${ }^{2}$ Priyanka Mocherla, ${ }^{2}$ Harry Jones, ${ }^{3}$ Jonas Hanisch, ${ }^{4}$ Thomas Bein, ${ }^{1}$ Piers R. F. Barnes, ${ }^{2}$ Pablo Docampo ${ }^{5, *}$

${ }^{1}$ Department of Chemistry and Center for NanoScience (CeNS), LMU Munich, Butenandtstr. 11, 81377 Munich, Germany

${ }^{2}$ Physics Department, Imperial College London, London, SW7 2AZ, United Kingdom

${ }^{3}$ Physics Department, University of Cambridge, Cambridge, CB3 0HE, United Kingdom

${ }^{4}$ Zentrum für Sonnenenergie- und Wasserstoff-Forschung (ZSW) Baden-Württemberg, Meitnerstr. 1, 70563 Stuttgart, Germany

${ }^{5}$ Physics Department, School of Electrical and Electronic Engineering, Newcastle University, Newcastle upon Tyne, NE1 7RU, United Kingdom

*Corresponding author: pablo.docampo@newcastle.ac.uk

Keywords: 2D perovskite, phase purity, crystal orientation, Ruddlesden-Popper phase, thin films, layered compound, solvent additive 


\section{Abstract}

Two-dimensional (2D) hybrid perovskites have attracted considerable attention due to their enormous structural and electronical variability, making this class of semiconductors interesting for photovoltaics, light-emitting diodes and lasers. 2D perovskites consist of sheets of bulky organic cations alternately sandwiched by layers of lead halide octahedra. The properties of these materials strongly depend on the thickness of the octahedra layers, defined by the number of octahedra sheets in a layer, $n$. Consequently, controlling the layer thickness purity (i.e. minimizing the spread in $n$ ) is important for any 2D perovskite thin film application. Here, we show that using rationally chosen solvent additives in the precursor solution offers a facile way to control the crystal disorder in 2D perovskites films. Our method leads to significantly reduced variation in $n$ around the target value relative to films obtained by conventional fast-crystallization methods without solvent additives. The improved phase purity in optimized $n=2$ and $n=3$ films is verified by X-ray diffraction, UV-Vis absorption, and photoluminescence measurements. Additionally, we find that 2D perovskite films with $n \geq 2$ arising from additive-assisted growth exhibit an unusual crystal orientation with the perovskite interlayers predominantly aligned parallel to the substrate, which we assign to the slow crystallization process induced by the lead-complexing solvent additives. Improved control over the phase purity translates into a better control of the optoelectronic properties of 2D perovskite films. Furthermore, the unusual horizontal crystal orientation of $n=2$ and $n=3$ films makes this family of tunable organic-inorganic perovskites promising for applications where lateral charge transport is desired, thus enlarging the potential for thin film-based applications of the 2D perovskites. 


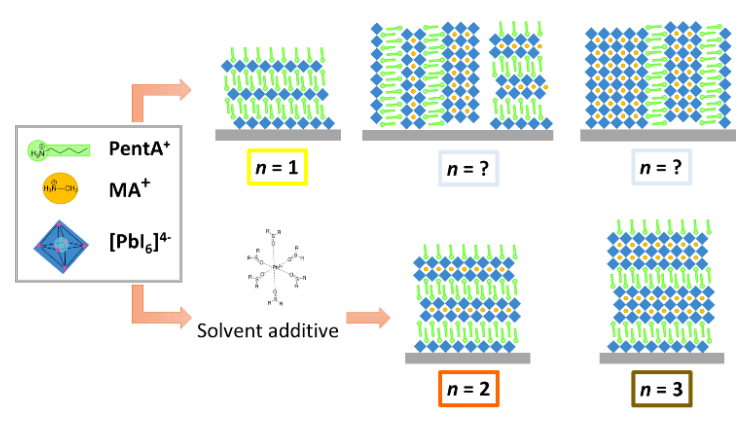

TOC graphic:

Improved phase purity in 2D hybrid perovskite thin films with horizontal crystal orientation was achieved through slow crystallization employing lead-complexing solvent additives.

\section{Introduction}

Two-dimensional (2D) hybrid perovskites, also often referred to as Ruddlesden-Popper perovskites, ${ }^{1,2}$ have recently attracted considerable interest for various potential semiconductor applications, such as solar cells, light-emitting diodes (LEDs) or lasers. ${ }^{3-9}$ This class of organicinorganic lead halide perovskite materials consists of alternating sheets of vertex-sharing lead halide octahedra, which can accommodate small organic cations such as methylammonium (MA), and interlayers of bulky ammonium-terminated organic cations. The long-chained organic cations (LOCs) usually feature alkyl chains or phenyl-groups, which can form bilayers via van-der-Waals or $\pi-\pi$ interactions, thus giving the $2 \mathrm{D}$ perovskite its periodic, layered crystal structure. ${ }^{10-12}$ In the field of photovoltaics, the replacement of 3D hybrid perovskites by their 2D analogues as photoabsorbers has led to substantially enhanced moisture stability of the resulting perovskite solar cells, a feature that has been attributed to the hydrophobic side chains of the incorporated LOCs. ${ }^{5,13-18}$ 
Although improving the moisture resistance of perovskite solar cells is important, the potential of 2D perovskites lies in their enormous structural tunability, which gives access to a large playground for tailoring their optoelectronic properties according to the application field. ${ }^{3-6,19}$ In particular, $n$, which is the number of octahedra sheets sandwiched between two organic interlayers, strongly determines the major features of the resulting 2D perovskite, such as bandgap, exciton binding energy or photoluminescence. ${ }^{13}$ 14, 19-23 A homologous series of Ruddlesden-Popper perovskite phases with the generic formula (LOC) $2(\mathrm{MA})_{n-1} \mathrm{~Pb}_{n} \mathrm{I}_{3 n+1}$ where $n=1,2,3,4$ and 5 has been successfully synthesized by Kanatzidis and co-workers, using butylammonium as the organic spacer. ${ }^{14,24}$ The fabrication of 2D perovskite single crystals with defined octahedra layer thickness $n$ was achieved by adjusting the ratio between the lead source, methylammonium iodide (MAI) and butylamine in the precursor solutions. The authors have shown that the bandgap of the $2 \mathrm{D}$ perovskites can be tuned from $2.4 \mathrm{eV}$ to $1.7 \mathrm{eV}$ by increasing the octahedra layer thickness from $n=1$ to $n=5$ respectively.

To obtain thin films of 2D perovskites with the desired $n$-value, conventionally the corresponding perovskite single crystals or a stoichiometric mixture of the precursor components is dissolved in $N, N$-dimethylformamide (DMF), followed by a spin-coating and annealing process. ${ }^{1,2,13,14,25}$ However, it has been largely overlooked that stoichiometry is not everything when it comes to the formation of 2D perovskite thin films on substrates. Thin films resulting from spin-coating of a stoichiometric 2D perovskite precursor solution are not necessarily composed of the targeted $n$ phase if careful control of the crystallization process is neglected. This is because the crystallization dynamics for thin film growth are significantly different from single crystal growth, not least because the nature of the substrate may influence the film nucleation and crystallization. 
Yet, controlling the phase purity regarding the octahedra layer thickness $n$ is imperative to obtain the desired optoelectronic properties of the 2D perovskite thin film.

In this work, we establish a simple fabrication method to obtain $n=2$ and $n=3$ perovskite films with minimized variation in $n$ from the target value. By using appropriate lead-complexing additives in the solvent and careful control of the annealing procedure, a narrow distribution of $n$ can be achieved in the resulting 2D perovskite film. By contrast, we demonstrate that nominally $n$ $=2$ and $n=3$ perovskite thin films spin-coated from DMF-solutions consist of a broad mixture of domains with different $n$-values when crystallized from traditional, fast-crystallization procedures. This often overlooked disorder is verified by temperature-dependent photoluminescence (PL) measurements, which reveals the presence of $n>3$ domains in a nominally $n=3$ perovskite film. We point out that rapid charge transfer from low- $n$ (2D-like) to high- $n$ (3D-like) domains in nonoptimized thin films at room temperature can be an explanation for the largely ignored discrepancy between the PL signal of $\mathrm{n} \geq 3$ perovskite films and the corresponding perovskite single crystals. Time-of-flight secondary ion mass spectrometry (ToF-SIMS) measurements confirm a gradient in $n$ within nominally $n=3$ perovskite films, with decreasing $n$-value from top to bottom. In addition to improved phase purity in the optimized 2D perovskite films reflected by a minimized variation in $n$, our controlled crystallization procedure results in $n=2$ and $n=3$ films with perovskite and organic interlayers that are oriented exclusively parallel to the substrate. This unusual crystal orientation not only allows us to unambiguously identify the perovskite thin films' $n$-value using X-ray diffraction measurements, but also makes the family of 2D perovskites interesting for device applications where lateral charge transport is required, such as back-contact solar cells or fieldeffect transistors. 


\section{Results and Discussion}

\section{Structural characterization}

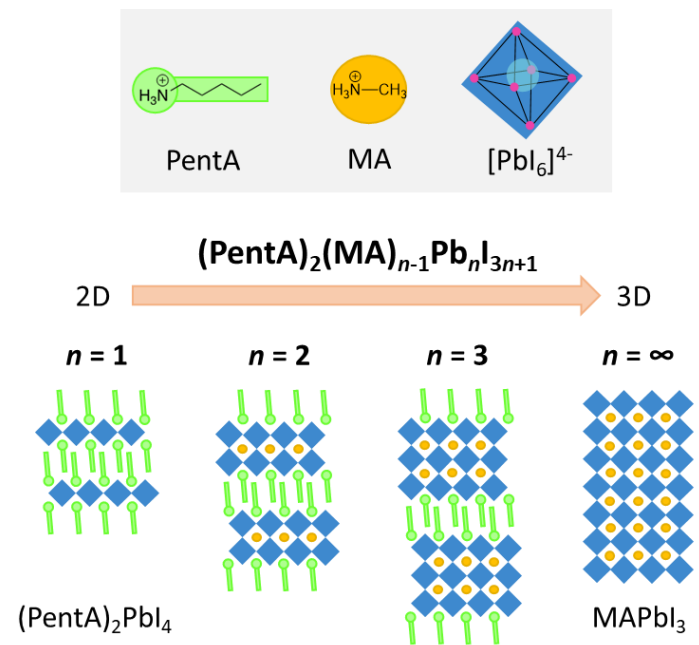

Figure 1. Schematic crystal structures of the homologous series of (PentA) $2(\mathrm{MA})_{n-1} \mathrm{~Pb}_{n} \mathrm{I}_{3 n+1}$ perovskites with the $n=1$ compound (PentA) ${ }_{2} \mathrm{PbI}_{4}$, intermediate MA-PentA mixed-cation 2D perovskites incorporating the $\mathrm{PbI}_{6}$ octahedra layers with thickness $n=2$ and $n=3$, and $\mathrm{MAPbI}_{3}$ as the $n=\infty$ case.

We investigated the homologous $(\mathrm{PentA})_{2}(\mathrm{MA})_{n-1} \mathrm{~Pb}_{n} \mathrm{I}_{3 n+1}$ series as a model system for $2 \mathrm{D}$ perovskites using pentylammonium (PentA) as the bulky organic cation. Figure 1 shows a schematic illustration of the layered perovskite structure composed of the following building blocks: bulky PentA cations, small MA cations and corner-sharing $\left[\mathrm{PbI}_{6}\right]^{4-}$ octahedra, where $n$ is the number of octahedra layers sandwiched between two PentA layers. For the case of $n=\infty$ (which occurs in the absence of PentA), the resulting crystal structure corresponds to the 3D perovskite methylammonium lead iodide $\left(\mathrm{MAPbI}_{3}\right)$. First, to fabricate thin films of the 2D perovskites, pentylammonium iodide (PentAI), methylammonium iodide (MAI) and $\mathrm{PbI}_{2}$ were mixed in the 
corresponding stoichiometry targeting a certain $n$-value and dissolved in DMF. Subsequently, the perovskite precursor solution was spin-coated on glass substrates in a one-step process following similar procedures as reported in literature. ${ }^{13,20,24}$ The $\mathrm{MAPbI}_{3}$ film was fabricated via an antisolvent drip method according to a previously established protocol. ${ }^{26}$ To characterize the crystal structure and the preferential orientation of the perovskite thin films, X-ray diffraction (XRD) measurements were performed. Figure 2 a shows the XRD patterns of non-optimized $n=1,2,3$ PentAMAPI films spin-coated from DMF-solutions and a $\mathrm{MAPbI}_{3}$ film.

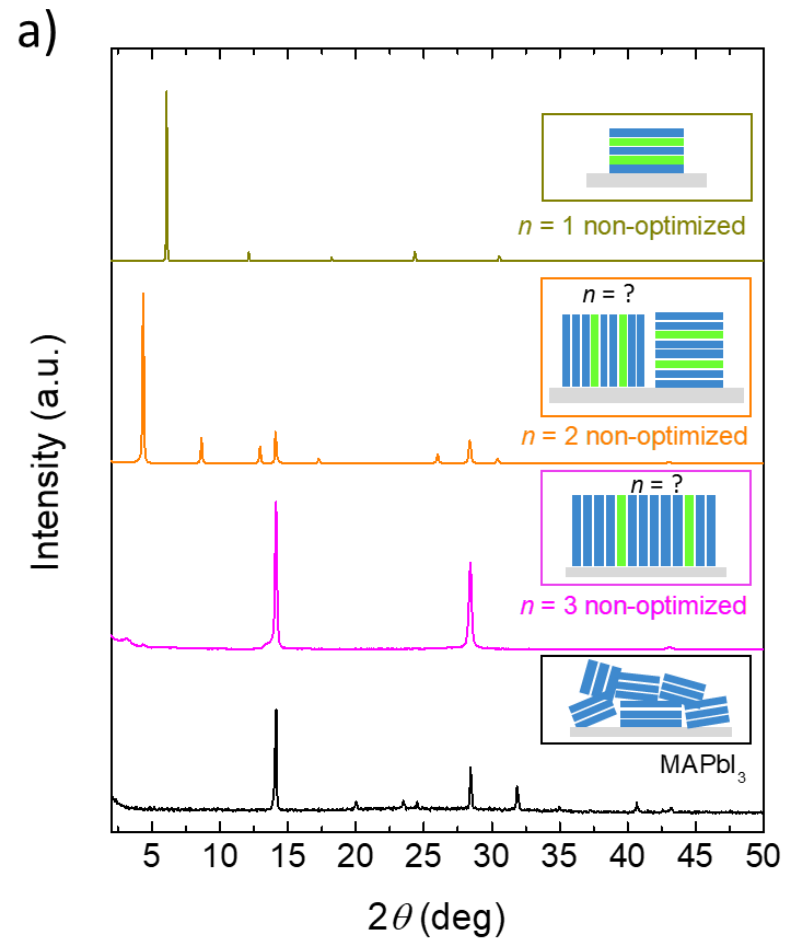

b)

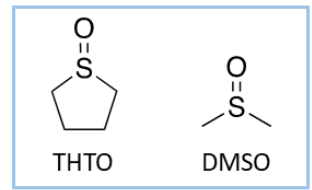

c)

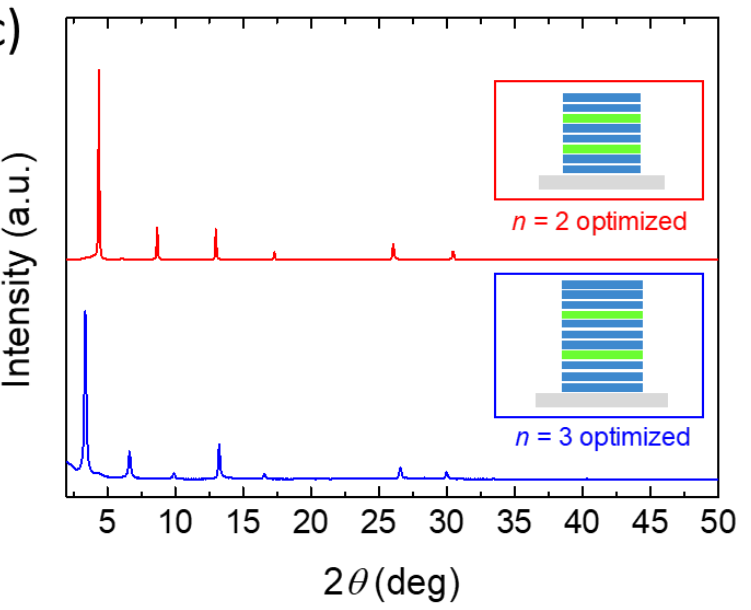

Figure 2. XRD patterns of (PentA) $)_{2}(\mathrm{MA})_{n-1} \mathrm{~Pb}_{n} \mathrm{I}_{3 n+1}$ perovskites: (a) non-optimized $n=1,2,3$ PentAMAPI films on glass substrates and a $\mathrm{MAPbI}_{3}$ film for comparison. (b) Solvent additives used for controlled 2D perovskite film growth and (c) the resulting optimized $n=2$ and $n=3$ PentAMAPI films. 
The presence or absence of certain diffraction peaks in XRD patterns of thin films recorded in the Bragg-Brentano scanning mode can give first indications for potentially preferred crystal orientation within the samples. Furthermore, the $2 \theta$ position of the diffraction peaks corresponding to the stacking direction of the perovskite interlayers reveals the dimensions of the unit cell and therefore the number of octahedra layers $n$. In our samples, the $n=1$ film exhibits pronounced peaks at the diffraction angle $2 \theta=6.1^{\circ}, 12.1^{\circ}, 18.2^{\circ}, 24.3^{\circ}$ and $30.5^{\circ}$, which can be indexed as the (002), (004), (006), (008) and (0010) reflections of the (PentA) $)_{2} \mathrm{PbI}_{4}$ phase. The high intensity of the diffraction peaks indicates very high crystallinity and the lack of other reflections can be assigned to a preferential orientation of the crystals along the $(00 l)$ direction.

This trend of growing layers which are oriented exclusively parallel to the substrate surface has been shown for the vast majority of $n=1$ Ruddlesden-Popper phase perovskites incorporating different ammonium-based cations. ${ }^{25,27-29}$ The XRD pattern of the $n=2$ film shows a similar set of diffraction peaks at $4.3^{\circ}, 8.6^{\circ}, 12.9^{\circ}$ and $17.3^{\circ}$, where the peak shift to smaller $2 \theta$ values corresponds to an increase in unit cell dimension from $14.5 \AA$ to $20.5 \AA$. As expected, this incremental increase in interplanar distance is roughly the same as the layer thickness of a sheet of vertex-sharing $\mathrm{PbI}_{6}$ octahedra. In addition, reflections at $14.1^{\circ}$ and $28.4^{\circ}$ can be ascribed to the (111) and (202) plane respectively. These two diffraction peaks occur when the alternating PentA and $\mathrm{PbI}_{6}$ interlayers are oriented perpendicular to the substrate. The co-existence of the (111) and (202) diffraction peaks and the low angle $(0 \mathrm{k} 0)$ peaks indicates that both horizontally and vertically oriented regions are present in the $n=2$ perovskite film, which agrees with previous reports., 14 
In contrast, the non-optimized, nominally $n=3$ film exclusively shows the (111) and the (202) reflection, which corresponds to the typical vertical orientation for high- $n$ members of the $2 \mathrm{D}$ perovskite phase. This vertical orientation of the interlayers has been reported for different 2D perovskite systems with a precursor stoichiometry of $n>2.1,13,17,24,30$ The resulting XRD patterns resemble the one of the 3D analogue $\mathrm{MAPbI}_{3}$, due to the same interplanar distance of $\sim 6.3 \AA$ which corresponds to the size of a $\mathrm{PbI}_{6}$ octahedron. However, the strong preferential crystal orientation where the perovskite interlayers are aligned perpendicular to the substrate does not allow an unambiguous determination of the $n$-value from the XRD pattern recorded in the conventional Bragg-Brentano mode, since information about the periodicity in the lateral direction (in-plane) is not revealed.

To complement our XRD results, which only provide us with out-of-plane reflections of these highly textured perovskite films, we used grazing-incidence wide-angle X-ray scattering (GIWAXS) techniques to examine all possible crystal orientations within the thin films. The GIWAXS pattern of the non-optimized $n=3$ film shows the (111) reflection at an azimuthal angle of $\chi=0^{\circ}$, verifying the exclusive vertical alignment of the perovskite interlayers with respect to the substrate (Figure S1a). Assuming that the $n=3$ periodicity of the 2D perovskite is present in the lateral direction, the corresponding diffraction peaks around the azimuthal angle $\chi=+/-90^{\circ}$ at low $q_{x y}$-values are expected to be present. However, the absence of this feature suggests crystal disorder in the lateral direction of the non-optimized, nominally $n=3$ film. Similar GIWAXS patterns have been shown for nominal $n=3,4$ or 5 films, which were identified as a mixture of multiple 2D perovskite phases with different $n$-values. ${ }^{24,31,32}$

Due to the close relationship between the $n$-value and the properties of the corresponding $2 \mathrm{D}$ perovskite, controlling its phase purity in thin films is vital for optoelectronic applications using 
this class of material. We demonstrate that the $n$-value phase purity can be significantly improved for $n=2,3$ PentAMAPI films by carefully controlling the crystallization process of the 2D perovskite film. This can be achieved by suitable solvent additives such as dimethyl sulfoxide (DMSO) or tetrahydrothiophene-1-oxide (THTO) in the precursor solution (see Figure $2 \mathrm{~b}$ ), combined with a slow annealing process during the perovskite film formation. Figure $2 \mathrm{c}$ shows the XRD patterns of the $n=2$ and $n=3$ PentAMAPI films prepared according to our optimized protocol using THTO and/or DMSO as solvent additives. Details about the optimized procedures are described in the methods section and summarized in the Supplementary Information (Table S1). We optimized the fabrication protocol with respect to spin-coater speed, additive concentration and annealing temperature to obtain 2D perovskite films showing a narrow distribution of $n$-values, close to the one targeted by the precursor stoichiometry. XRD was employed to monitor the optimization process for $n=2$ and $n=3$ PentAMAPI (see Supplementary Information, Figure S2 and Figure S3) on glass substrates.

Compared to the non-optimized $n=2$ and $n=3$ films, the optimized 2D perovskite films exhibit strong $(0 k 0)$ peaks for both samples. The presence of THTO in the $n=2$ precursor solution leads to a significant increase in overall diffraction peak intensities (Figure S2a), verifying higher crystallinity of the $n=2$ film grown with the additive-assisted approach. In particular, the diffraction peaks at $3.3^{\circ}, 6.6^{\circ}, 9.9^{\circ}$ and $13.2^{\circ}$ for the optimized $n=3$ film show that, in addition to the high crystallinity, the perovskite interlayers exhibit an unusual orientation which is parallel to the substrate (Figure 2c). The "horizontal" orientation of the perovskite layers for $n=3$ is verified by the corresponding GIWAXS pattern (Figure $\mathrm{S} 1 \mathrm{~b}$ ), where the $(0 k 0)$ signals do not form diffraction rings, but are exclusively found at an azimuthal angle $\chi=0^{\circ}$. In contrast to the vertically oriented, non-optimized (nominally) $n=3$ perovskite film, this allows us to unambiguously 
confirm the octahedra layer thickness to be $n=3$ from the $2 \theta$ position of the $(020)$ reflection in the XRD pattern. The absence of the (111) and (202) reflections in the XRD pattern indicates that vertical growth is largely suppressed in both optimized $n=2$ and $n=3$ films. To the best of our knowledge, this is the first demonstration of predominantly horizontally oriented 2D perovskite thin films with $n>1$. The recipe for the $n=1$ samples and our optimized protocols for $n=2$ and $n=3$ films were tested on different substrates, including fluorine-doped tin oxide (FTO), FTO/ $\mathrm{TiO}_{2}$, indium-doped tin oxide (ITO), and silicon. The XRD patterns shown in Figure S4 demonstrate that the 2D perovskite phase purity and crystal orientation is comparable for all tested substrates.

We note that a slow annealing procedure at a lower starting temperature is vital to obtain $2 \mathrm{D}$ perovskite films that satisfy phase purity. The best results were achieved by following a three-step drying sequence, with a stepwise increase of the annealing temperature from $40^{\circ} \mathrm{C}$ to $75^{\circ} \mathrm{C}$ to 100 ${ }^{\circ} \mathrm{C}$ (see Supplementary Information, Table S1). Without this stepwise annealing less uniformity was achieved: if $n=3$ films, including DMSO as a precursor additive, were prepared by directly heating at $100{ }^{\circ} \mathrm{C}$ after spin-coating, the XRD pattern of the resulting film showed diffraction peaks from a mixture of horizontally oriented $n=1,2$ and 3 phases, indicating poor phase purity (Figure $\mathrm{S} 3 \mathrm{c})$.

\section{Film morphology}

Scanning electron microscopy (SEM) top view images for the $n=1$ and 2 samples can be found in Figure S5. Anisotropic growth of pure 2D perovskite crystals is visible in the formation of platelike single-crystals for low $n$-values, as previously reported. ${ }^{24}$ This is also reflected in the 
morphology of the crystallites within the $n=1$ film, which consists of elongated crystals with a preferential growth direction (likely along the plane of the organic layer). For the $n=2$ and $n=3$ films, the increasing number of MA promotes a more isotropic growth, which results in more spherically shaped crystallites.

SEM top view images of the non-optimized and optimized $n=3$ film reveal similar crystal morphologies with a grain size around $50 \mathrm{~nm}$ (Figure 3a-b). Encouragingly, the optimized $n=3$ film exhibits significantly fewer cracks on the sample surface and the corresponding atomic force microscopy $(\mathrm{AFM})$ images indicate significantly reduced root-mean-square film roughness $\left(r_{\mathrm{rms}}=\right.$ $14 \mathrm{~nm}$ ) compared to the non-optimized sample without solvent additives $\left(r_{\mathrm{rms}}=25 \mathrm{~nm}\right)$, as shown in Figure $3 \mathrm{c}-\mathrm{d}$. The slower, more controlled crystallization of the optimized $n=3$ films in the presence of DMSO prevents the formation of large cracks and supports the formation of more uniform and continuous films compared to non-optimized films obtained from traditional fast crystallization methods. The same trend has been previously shown for MAPI films, which underlines the importance of the annealing temperature for the fabrication of high-quality perovskite films. The good film quality of the optimized 2D perovskite thin films is promising for various device applications, which require uniform layers to support sufficient charge transport. However, the relatively small grain size of $\sim 50 \mathrm{~nm}$ for the $2 \mathrm{D}$ perovskites may create some limitations. Larger crystal grains are in general more desirable due to fewer boundaries that can impede effective charge transport. To eliminate grain size as limiting factors for device performance, we are currently investigating attempts to increase the grain size (e.g. by solvent annealing) without disturbing the phase purity and crystallite orientation. 

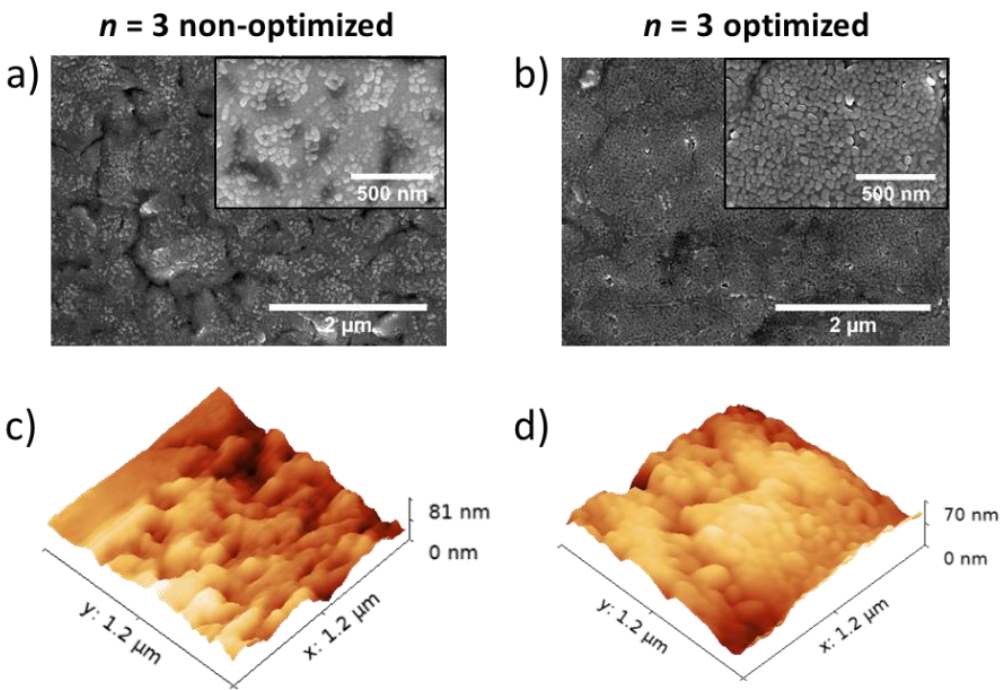

Figure 3. SEM top view images of the (a) non-optimized and (b) optimized $n=3$ perovskite film with solvent additives prepared on $\mathrm{FTO} / \mathrm{TiO}_{2}$ substrates. The insets show $\mathrm{SEM}$ images at higher magnification. AFM images show the surface roughness of the (c) non-optimized and (d) optimized $n=3$ perovskite film.

\section{Optical characterization}

Since the optoelectronic properties of $2 \mathrm{D}$ perovskites strongly depend on $n$, our structural analysis employing XRD techniques was complemented by optical characterization to obtain further insights into the phase purity of 2D perovskite thin films. Figure 4a shows the normalized UV-Vis absorption profiles of non-optimized $n=1,2$ and 3 perovskite films spin-coated from DMF solutions and a $\mathrm{MAPbI}_{3}$ film for comparison. In agreement with previous reports, the absorption onsets of the 2D perovskites are substantially shifted to longer wavelengths with increasing $n$, 
which is apparent as a change of the film's color (see inset Figure 4a). ${ }^{2,21}$ The $n=1$ (yellow), 2 (red) and 3 (brown) films show distinct excitonic features at $488 \mathrm{~nm}, 563 \mathrm{~nm}$ and $605 \mathrm{~nm}$, respectively. ${ }^{14}$ The non-optimized $n=3$ film also shows the signature of the $n=2$ phase and vice versa, hinting at a mixture of phases in both samples. In addition, the non-optimized nominally $n$ $=3$ sample exhibits an absorption tail up to $740 \mathrm{~nm}$, which can be interpreted as an indication for the presence of $n>3$ crystal regions with a narrower bandgap than the nominal $n=3$ phase, similar to the $\mathrm{MAPbI}_{3}$ film $(n=\infty)$ with a bandgap of $1.6 \mathrm{eV}$ and an absorption onset around $770 \mathrm{~nm}$. By comparison, the optimized $n=3$ film shows a less pronounced absorption tail (Figure $4 \mathrm{c}$ ) and a reduced $n=2$ absorption peak, giving evidence for a more narrow distribution in $n$. The estimated bandgaps for the optimized $n=1,2$ and 3 films are 2.3, 2.2 and $2.0 \mathrm{eV}$ respectively, which is in agreement with the bandgap values reported for the corresponding $2 \mathrm{D}$ perovskite single crystals. ${ }^{2}$ 
a)

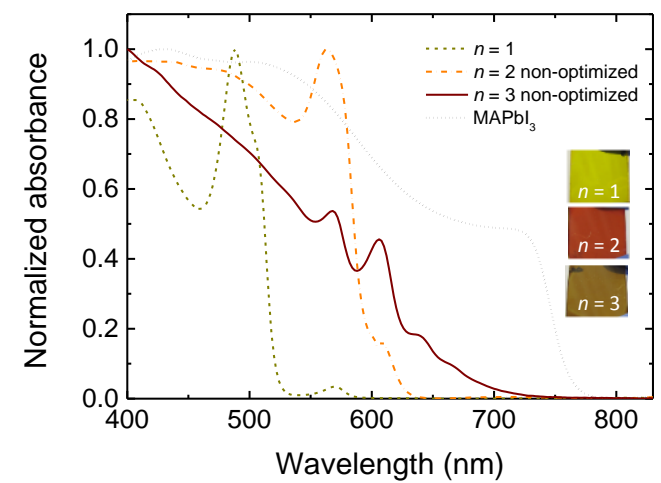

c)

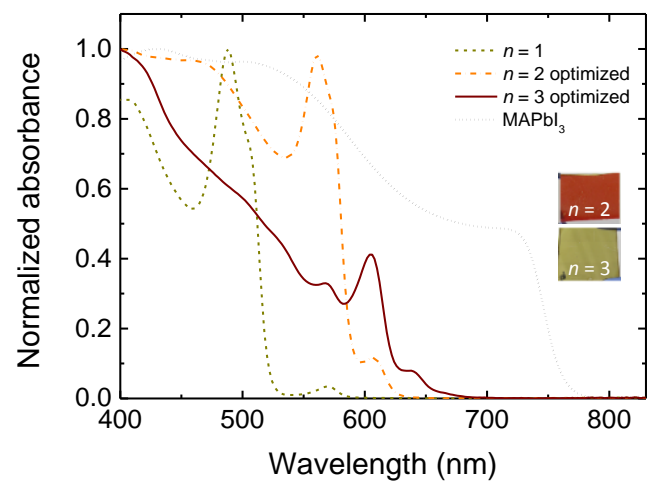

b)

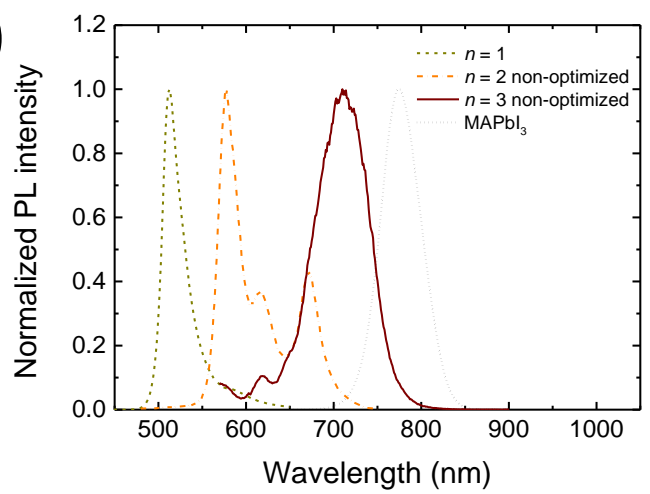

d)

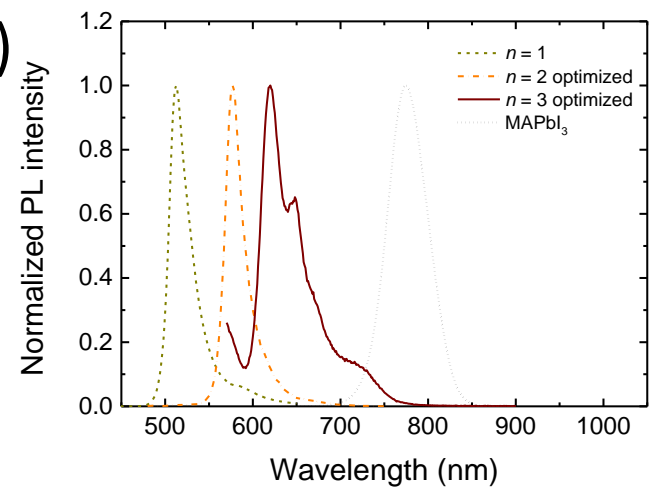

Figure 4. Normalized UV-Vis absorption spectra and PL spectra of non-optimized (a-b) and optimized (c-d) 2D perovskite films with $n=2$ and $n=3$ on glass compared to $n=1$ and $\mathrm{MAPbI}_{3}$ films.

Furthermore, we conducted photoluminescence (PL) measurements to evaluate the distribution of $n$ within the 2D perovskite and potential charge transfer between these crystal domains. In Figure $4 \mathrm{~b}$, the room temperature steady-state PL spectra of a $\mathrm{MAPbI}_{3}$ film and the non-optimized $n=1$, 2 and 3 perovskites films are displayed. In agreement with literature values, the extreme cases $n=$ 1 and $\mathrm{MAPbI}_{3}(n=\infty)$ both show a single PL peak around $524 \mathrm{~nm}$ and $776 \mathrm{~nm}$ respectively, confirming the phase purity of the perovskite films. ${ }^{2,33}$ The non-optimized $n=2$ film shows the 
expected PL peak at $570 \mathrm{~nm}$, accompanied by two additional peaks at $625 \mathrm{~nm}$ and $675 \mathrm{~nm}$, which can be assigned to the $n=3$ and $n=5$ phase., ${ }^{2,24}$

Interestingly, the non-optimized $n=3$ film exhibits only a minor peak at $625 \mathrm{~nm}$, which coincides with the PL signature of $n=3$ single crystals, ${ }^{2}$ while the main PL feature consist of a broad peak with a maximum around $720 \mathrm{~nm}$. This is consistent with all previous reports about $n \geq 3$ perovskite films, independent of the nature of the bulky organic cation or the film fabrication method. . $^{13,14}$ 20, 24 The discrepancy between the PL spectra of 2D perovskite singles crystals and the corresponding thin films fabricated from DMF solutions without solvent additives indicates a large variety of $n$ within these perovskite films which are likely to result from rapid crystallization. Moreover, photoemission in these non-optimized 2D perovskite films seems to be dominated by recombination events from layers where $n>>3$.

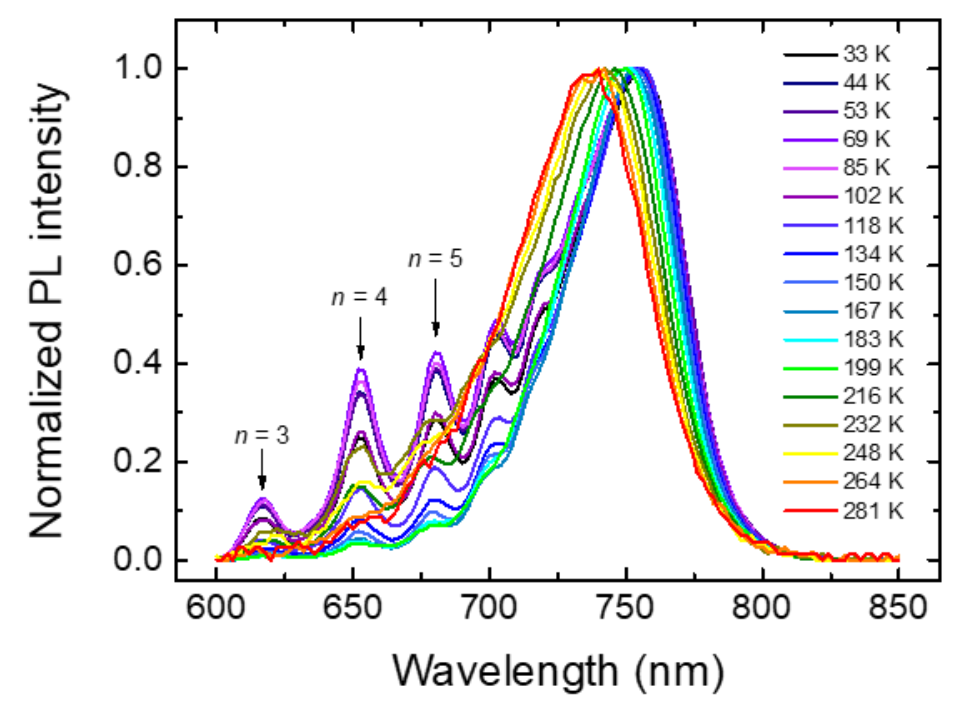

Figure 5. Normalized temperature-dependent PL spectra of a non-optimized $n=3$ PentAMAPI film on glass recorded in the temperature range 33-281 K. The characteristic PL peaks of the $n=$ 3,4 and 5 perovskite phases occurring at low temperatures are indicated with arrows. 
To understand the origin of the observed emission from the large- $n$ domains, we performed temperature-dependent PL measurements on non-optimized $n=3$ perovskite films (Figure 5). Interestingly, after cooling down the sample to $30 \mathrm{~K}$, several distinct PL peaks emerge, which can be attributed to the presence of $n=3,4,5$ regions, underlining the proposed presence of phase impurities in non-optimized films. As low temperatures, the activation energy for efficient charge transfer between domains with different $\mathrm{n}$ cannot be overcome thermally. The broad distribution of $n$-values within the non-optimized 2D perovskite film is therefore unambiguously reflected in the PL signal. As the temperature is gradually raised to $298 \mathrm{~K}$, the distinct PL features of the low$n$ regions diminish, while the emission from the bulk-like large- $n$ areas around $720 \mathrm{~nm}$ steadily increases. Assuming that structural changes such as phase transformation between the different regions do not occur, our results suggest that the observed single PL peak of non-optimized $n=3$ films can be rationalized by thermally activated transport and relaxation of charge carriers from crystal regions of wide bandgap $(n \leq 3)$ to regions where the bandgap approaches the bulk perovskite material $(n>>3)$. This is in accordance with recent reports by Liu et al.,$^{32}$ confirming rapid exciton transport from low- $n$ regions to large- $n$ regions on the picosecond time scale at room temperature. The fast transport of photogenerated carriers leads to radiative recombination from solely the high- $n$ regions and conceals the presence of low- $n$ phases. The apparent single PL peak for various 2D perovskite films obtained from fast crystallization has been repeatedly misinterpreted as an evidence for phase purity, despite the obvious deviation from single crystal PL.

In comparison, the PL spectra of the optimized $n=2$ and $n=3$ films using THTO and/or DMSO as solvent additives show one predominant peak at $570 \mathrm{~nm}$ and $625 \mathrm{~nm}$ respectively (Figure $4 \mathrm{~d}$ ). 
These PL peaks match well with the PL profile of the corresponding $n=2$ and $n=3$ single crystals, as reported by Stoumpos et al. ${ }^{2}$ In particular, the PL signal of the optimized $n=3$ film exhibits only a small additional peak at $650 \mathrm{~nm}(n=4)$ and a shoulder around $725 \mathrm{~nm}(n>>3)$. Therefore, we conclude that the addition of DMSO or THTO and careful control of the annealing process result in 2D perovskite films with significantly less variation in $n$ from the target value than films prepared without the solvent additives, which is in agreement with our XRD results.

\section{Depth profile analysis}

Despite the improved phase purity of the additive-assisted formation of $n=3$ PentAMAPI perovskite thin films regarding $n$, our PL analysis indicates that some large- $n$ regions remain within the optimized films. In order to assess the spatial distribution of the different $n$-regions, time-offlight secondary ion mass spectrometry (ToF-SIMS) measurements were performed. Since the $n$ value is determined by the ratio between MA and PentA cations, a comparison of the depth profiles of the $\mathrm{MA}^{+}$and $\mathrm{PentA}^{+}$species allows us to estimate the vertical distribution of the large- $n$ regions. 


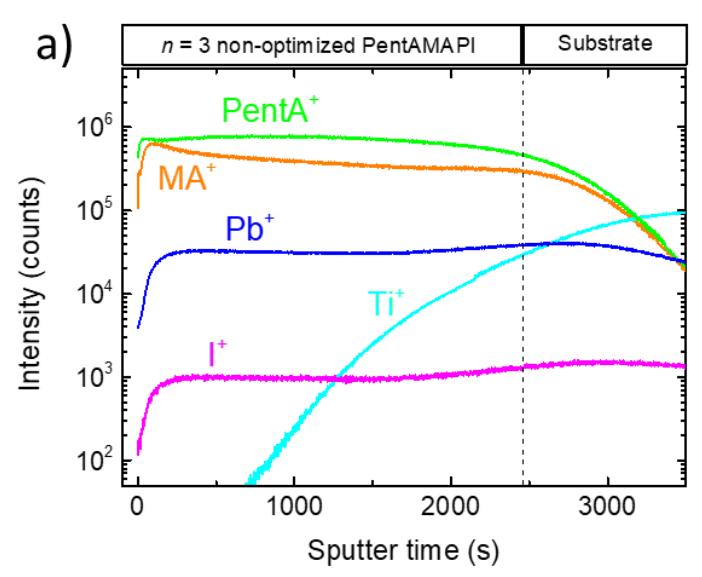

c)

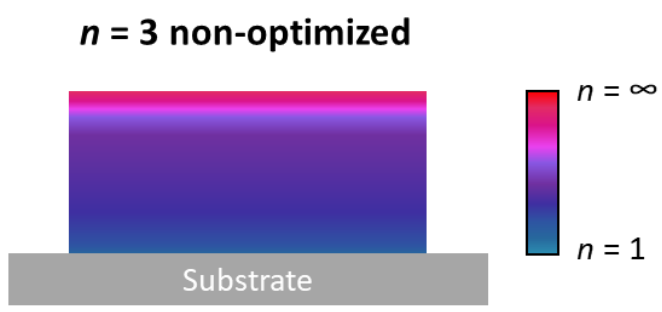

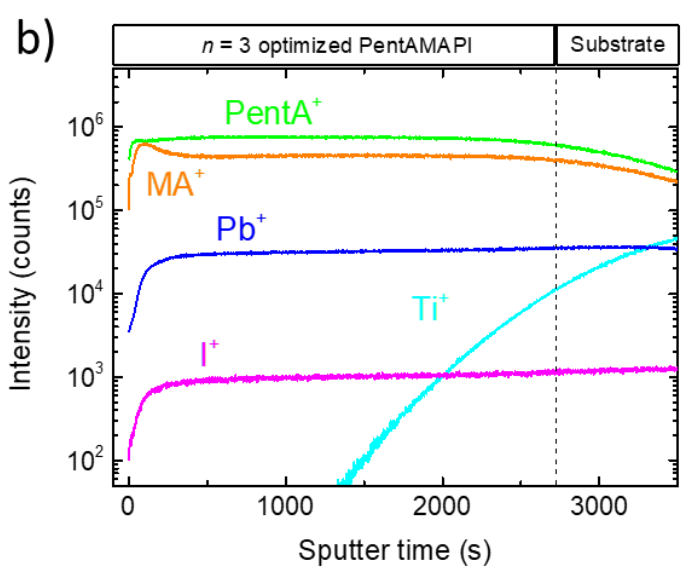

d)

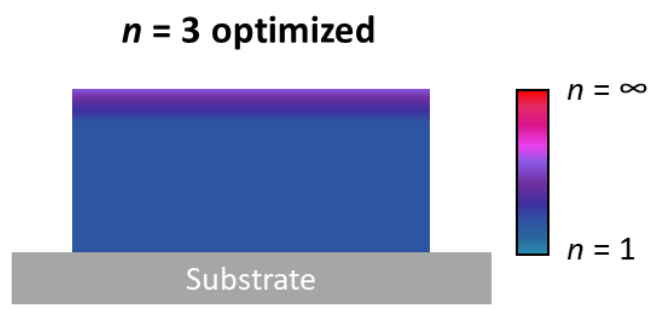

Figure 6. Positive ToF-SIMS depth profiles of (a) non-optimized and (b) optimized $n=3$ perovskite film on $\mathrm{FTO} / \mathrm{TiO}_{2}$ substrates. The dotted line is a guide to the eye for the estimated interface between perovskite and $\mathrm{TiO}_{2}$. Proposed vertical distribution of crystal regions with different $n$-values for (c) non-optimized and (d) optimized $n=3$ films.

Figure $6 \mathrm{a}-\mathrm{b}$ shows the positive ToF-SIMS depth profiles of the non-optimized and the optimized $n=3$ films prepared on $\mathrm{FTO} / \mathrm{TiO}_{2}$ substrates. Similar trends are observed for both samples: the vertical distribution of Pent $\mathrm{A}^{+}$cations is rather constant throughout the perovskite film, whereas a notable enrichment of $\mathrm{MA}^{+}$species is detected at the beginning of the sputtering process, i.e. at the film's surface. We interpret this observation as an indication for the formation of large- $n$ regions on the very top of the perovskite film for both non-optimized and optimized $n=3$ samples, with a gradient of decreasing $n$ towards the bottom (Figure $6 \mathrm{c}-\mathrm{d}$ ). This finding is in excellent 
agreement with recent studies by Liu et al. and Shang et al. ${ }^{31,32}$ We note that the $\mathrm{MA}^{+}$depth profile for the optimized sample varies less after the initial rise than for the non-optimized sample. As evidenced by XRD and PL experiments, we observe a significantly improved phase purity for optimized $n=3$ films. We therefore propose that the main phase is $n=3$, as desired, with a small fraction of $n>3$ phases at the sample surface.

\section{Proposed mechanism for perovskite film formation}

Having identified several factors which affect the disparity in crystal disorder and orientation in 2D perovskite thin films, such as the precursor stoichiometry, the solvent and the annealing temperature, we need to understand how these factors relate to each other. Such knowledge may enable us to control the formation process of 2D perovskite films. Herein, we attempt to rationalize the large variation in $n$ and the changes in crystal orientation, from horizontal to vertical, in 2D perovskite thin films, caused by changes in precursor stoichiometry without adjusting the solvent. Moreover, we propose a simple film formation mechanism that offers a possible explanation for the role of the solvent additives and the annealing temperature in achieving an improved phase purity of 2D perovskite layers. 
a)
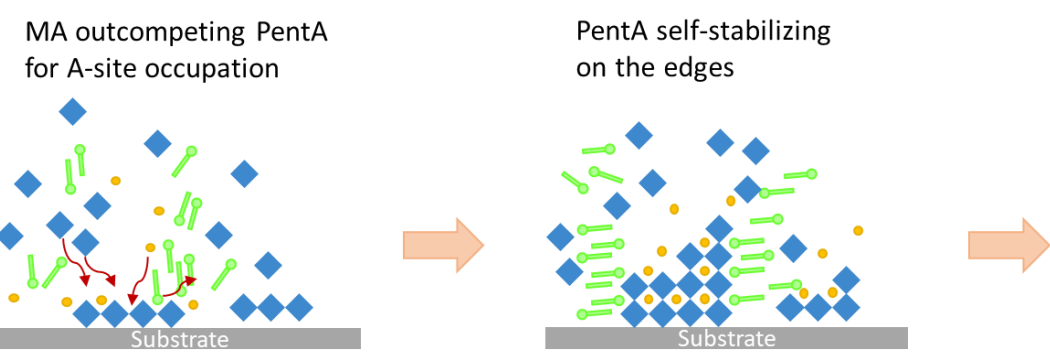

Fast vertical growth

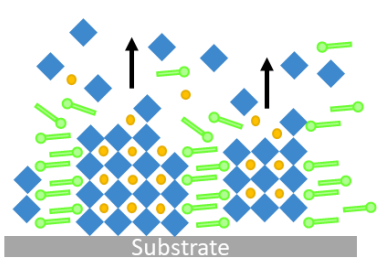

b) DMSO complexes $\mathrm{Pb}^{2+}$

MA not outcompeting PentA
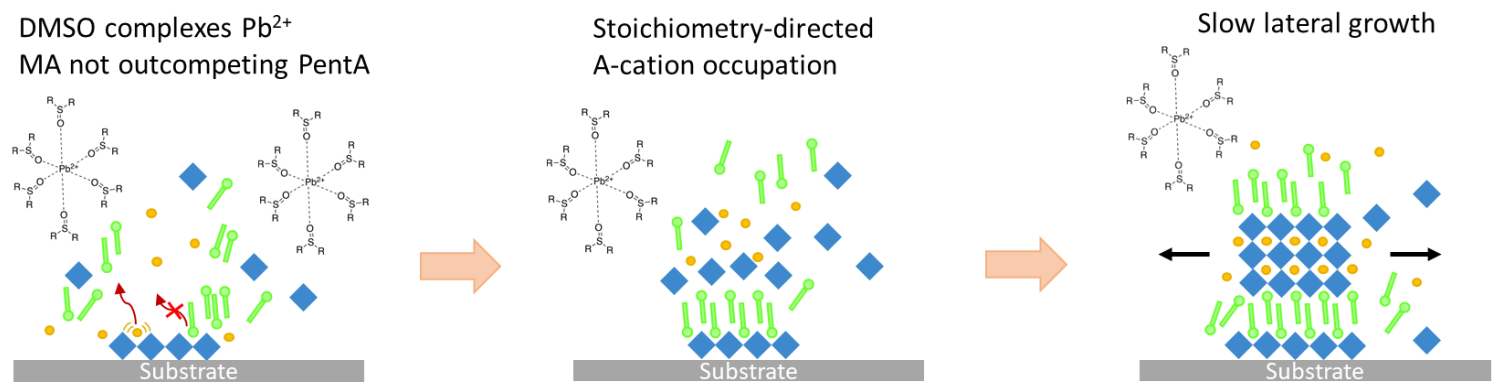

Figure 7. Schematic illustration of the hypothesized formation mechanism for a) non-optimized $n$ $=3$ films via fast vertical growth and $\mathrm{b}$ ) optimized $n=3$ films via solvent additive-assisted slow lateral growth.

Figure 7a shows a schematic illustration of the proposed crystallization mechanism for the nonoptimized DMF-based $n=3$ film. First, we assume that heterogeneous nucleation commences with the formation of $\mathrm{PbI}_{6}$ octahedra clusters at the liquid-substrate interface. This assumption can be justified by the strongly suppressed formation of any crystalline phase (neither PentAI nor MAI) in a spin-coated, non-annealed 2D perovskite film (e.g. $n=2)$ when lead ions are withheld by THTO as a solvent additive (Figure S6). In the next step, a competition between MA and PentA cations occurs since these two species both possess a positively charged ammonium group to bind to the exposed A-site on top of the $\mathrm{PbI}_{6}$ octahedra. Despite the 1:1 ratio of MA and PentA, it is likely that MA outcompetes the PentA cations and successfully binds to the favorable A-site due 
to its smaller ionic radius and stronger dipole moment. The small MA cations can then be quickly "locked-in" by free $\mathrm{PbI}_{\mathrm{x}}$ clusters, which are abundant in the DMF precursor solution. At the same time, the facile evaporation of the DMF solvent induces fast vertical growth of perovskite layers, whereas the PentA cations are pushed to the edge of the growing perovskite "wall", stabilizing themselves via van-der-Waals forces between the alkyl chains and thus forming the organic bilayer. For $n>2$, this rapid vertical growth seems to dominate, which could explain the broad distribution of octahedra layer thicknesses.

When DMSO or THTO is added to the precursor solution, the kinetics of the film growth change dramatically. It has been shown by Foley et al. that the sulfoxide group of DMSO and particularly THTO strongly interact with $\mathrm{Pb}^{2+}$ ions in the solution, forming stable complexes. ${ }^{34,35}$ The authors demonstrated that THTO slows down the crystallization process of $\mathrm{MAPbI}_{3}$, which leads to an unusual (100) crystal orientation in the resulting 3D perovskite film. In the case of 2D perovskites, this interaction between solvent additive and $\mathrm{Pb}^{2+}$, in combination with a slow annealing process at low temperatures, presumably has two major effects on the growth dynamics of the $2 \mathrm{D}$ perovskite film, as schematically illustrated in Figure $7 \mathrm{~b}$ :

(i) $\mathrm{Pb}^{2+}$ is withheld in the liquid phase in the form of sulfoxide-complexes, thus reducing the amount of available $\mathrm{PbI}_{\mathrm{x}}$ clusters, meaning that the MA cations cannot be immediately "lockedin" after occupying the A-sites. This might allow the large PentA cations to compete with the smaller MA cations for the preferred top A-site, thereby forming organic PentA bilayers parallel to the substrate and resulting in horizontal growth of the perovskite interlayers as well. Increasing the sulfoxide content leads to more $\mathrm{Pb}^{2+}$ complexation, thus decelerating the vertical growth induced by MA cations. This is supported by the increased XRD peaks of the horizontally oriented 
2D perovskite phase and decreased reflections of the vertical phase with increased DMSO or THTO content as shown in Figure S2a and Figure S3b.

(ii) The slow, controlled horizontal growth at low initial annealing temperatures enables the stoichiometry between MA and PentA to define the final layer thickness of the perovskite sheet.

Our results show that this leads to $2 \mathrm{D}$ perovskite films which show substantially less variation in $n$ from the target value than films grown from a fast crystallization process through immediate annealing at high temperatures.

However, we note that our current choice of solvent additives and perovskite precursors does not result in phase pure and horizontally oriented $n=4$ films. We observe that the vertical alignment of the perovskite interlayers remains dominating in spite of an increase in DMSO additive volume, while the addition of THTO leads to the formation of a mixture of low- $n$ phases (Figure S7). We suspect that the large amount of MA compared to PentA in the $n=4$ stoichiometry allows MA to outcompete PentA for the A-binding site even in the presence of $\mathrm{Pb}$-complexing additives. Therefore, we are currently exploring different cation mixtures to control the binding of the small A-cation and the bulky organic cation in order to stabilize 2D perovskite phases with $n>3$.

\section{Implication for potential device applications}

Minimizing the variation in $n$ within 2D perovskite films is not only important from a theoretical point of view, but it can also have a profound impact on device performance. The potential migration of mobile charge carriers in the mixed-phase 2D perovskite layer to low- $n$ regions might result in a lower open-circuit voltage in perovskite solar cells than expected from a high-bandgap 2D perovskite material. In perovskite-based light emitting diodes (LEDs), this charge transfer in mixed-phase 2D perovskite layer might result in the requirement for a greater driving overpotential 
relative to the energy of emitted photons. Hence, approaches to control the disorder within 2D perovskites will have significant technological importance.

Another important aspect arising from the structural anisotropy of 2D perovskites is their crystal orientation when fabricated as thin films. To date, conventional preparation methods for thin films of 2D perovskite phases formed from $n>2$ solutions result in layers oriented perpendicular to the substrate. For photovoltaic applications, this is considered to be an advantage since the standard solar cell architectures require transport of charge carriers perpendicular to the substrate. Charge transport is likely to be confined within the perovskite component of the layers, since the addition of LOC interlayers oriented perpendicularly to the charge transport direction leads to a notable drop in current. ${ }^{36}$

However, there are many potential applications of 2D perovskites where lateral charge transport would be beneficial, for example in a back-contact solar cell or a field-effect transistor architecture. ${ }^{37-40}$ In this case, conducting perovskite layers which are oriented parallel to the substrate might also have a potential advantage of inhibiting the migration of ionic defects to or from the gate electrode, which might reduce screening of the gate voltage by ionic rather than electronic charge. By establishing a facile additive-assisted fabrication method to control the orientation of the perovskite layers, our work considerably enlarges the potential thin film-based application fields for $2 \mathrm{D}$ perovskites.

\section{Conclusions}

In conclusion, we point out that 2D Ruddlesden-Popper perovskite films obtained from traditional fast-crystallization using DMF-based stoichiometric precursor solutions with $n \geq 2$ are generally 
not phase-pure materials, but a mixture of domains with different $n$. The vertical orientation of the perovskite interlayers with respect to the substrate for nominally $n>2$ films makes it difficult to assess real distribution in $n$ with X-ray diffraction methods. Therefore, optical characterization techniques which unambiguously reveal the fingerprints of perovskite phases with different $n$ are indispensable to complement the structural analysis and to identify the phase purity of 2D perovskite thin films. In this work, we established a simple fabrication method using rationally chosen lead-complexing solvent additives for $2 \mathrm{D}$ perovskite films to substantially reduce the variation in the thickness $n$ of the lead halide octahedra interlayers from the target value. Furthermore, we demonstrate that for the specific $n=3$ case, our optimized films show similar PL features as reported for $n=3$ perovskite single crystals. By contrast, the PL signal of films resulting from fast-crystallization without solvent additives is largely dominated by photoemission from perovskite interlayers with $n>>3$, indicating rapid charge transfer from low- $n$ domains to large- $n$ domains. We proposed a simple crystallization mechanism as an empirical explanation for the poor phase purity in films obtained from fast-crystallization, and for the reduction in crystal disorder through slow additive-assisted growth. The improved control over the phase purity by retarding the crystal growth is crucial for controlling the fundamental optoelectronic properties of 2D perovskite thin films. In addition, our solvent engineering approach leads to an unusual, predominantly horizontal crystal orientation in the $n=2$ and $n=3$ perovskite films. This opens the doorway for a wide range of potential applications of 2D perovskites that require lateral charge transport.

\section{Methods}


Synthesis of PentAI. PentAI crystals were synthesized by adding dropwise $15 \mathrm{~mL} \mathrm{HI} \mathrm{(57} \mathrm{wt \%} \mathrm{in}$ water, in-house supplier) to a mixture of $13.5 \mathrm{~mL}$ 1-pentylamine (99\%, Sigma-Aldrich) and $100 \mathrm{~mL}$ ethanol (absolute, in-house supplier) under ice-cooling and vigorous stirring. The solution was stirred for $30 \mathrm{~min}$ at room temperature. After removal of the solvent by rotary evaporation at $50{ }^{\circ} \mathrm{C}$, the white precipitate was redissolved several times in ethanol and recrystallized from dry isopropanol (in-house supplier). The obtained colorless crystals were filtered, washed with diethyl ether (anhydrous, Sigma-Aldrich) and dried in vacuum for $4 \mathrm{~h}$.

Precursor Solutions. All steps of the preparation of the perovskite precursor solutions and the thin films were conducted in a glove box under dry nitrogen atmosphere. Using the generic chemical formula for the 2D hybrid lead halide perovskites (PentA) $)_{2}(\mathrm{MA})_{n-1} \mathrm{~Pb}_{n} \mathrm{I}_{3 n+1}$, the stoichiometry of the precursors PentAI, MAI (Dyesol) and $\mathrm{PbI}_{2}$ (99.99\%, TCI) was determined for octahedra interlayer thicknesses of $n=1,2$ or 3. The PentAI:MAI:PbI 2 ratios were 2:0:1 mmol, 1:0.5:1 mmol, 0.4:0.4:0.6 mmol and 0.333:0.333:0.5 mmol for $n=1,2$, non-optimized $n=3$ and optimized $n=3$ respectively, in $1 \mathrm{~mL}$ of DMF (anhydrous, Sigma-Aldrich). Table S1 in the Supplementary Information summarizes the net weights for the different components used for a certain target value of $n$. The precursors were dissolved at $100{ }^{\circ} \mathrm{C}$, the bright yellow solution was cooled to room temperature and filtered through a $0.45 \mu \mathrm{m}$ syringe filter. As solvent additives, $100 \mu \mathrm{L}$ of THTO (97\%, Sigma-Aldrich) and $100 \mu \mathrm{L}$ DMSO (anhydrous, Sigma-Aldrich) were added to $1 \mathrm{~mL}$ of the DMF-based $n=2$ precursor solution. Similarly, $350 \mu \mathrm{L}$ DMSO was added to $1 \mathrm{~mL}$ of the $n=3$ precursor solution for the optimized recipe. The stoichiometric $\mathrm{MAPbI}_{3}$ solution contained $1.25 \mathrm{mmol}$ of $\mathrm{PbI}_{2}$ and MAI respectively, dissolved in a mixture of $800 \mu \mathrm{L}$ DMF and $200 \mu \mathrm{L}$ DMSO.

Thin Film Fabrication. For $n=1,2$ and non-optimized $n=3$ films, $50 \mu \mathrm{L}$ of the respective precursor solution was dynamically spin-coated on a plasma-cleaned glass substrate $(2.5 \times 2.5 \mathrm{~cm})$ at $3000 \mathrm{rpm}$ for $40 \mathrm{~s}$. The optimized $n=3$ films were spin-coated at $4000 \mathrm{rpm}$. Afterwards, the substrate was annealed on a hotplate to evaporate residual solvents and to further promote crystallization. The $n=1$ and the non-optimized $n=2$ and 3 films were annealed at $100{ }^{\circ} \mathrm{C}$ for 5 $\min$. The optimized $n=2,3$ films were annealed first at $40{ }^{\circ} \mathrm{C}$ for $30 \mathrm{~min}$, then at $75{ }^{\circ} \mathrm{C}$ for $5 \mathrm{~min}$ and finally at $100{ }^{\circ} \mathrm{C}$ for $2 \mathrm{~min}$. $\mathrm{MAPbI}_{3}$ was spin-coated in a two-step program at $1000 \mathrm{rpm}$ and $5000 \mathrm{rpm}$ for $10 \mathrm{~s}$ and $30 \mathrm{~s}$ respectively. $500 \mu \mathrm{L}$ chlorobenzene (anhydrous, Sigma-Aldrich) were 
added as an anti-solvent to the spinning film at $15 \mathrm{~s}$ before the end. The $\mathrm{MAPbI}_{3}$ sample was annealed at $40{ }^{\circ} \mathrm{C}$ for $40 \mathrm{~min}$ and finally at $100{ }^{\circ} \mathrm{C}$ for $10 \mathrm{~min}$.

Characterization. XRD measurements were carried out with a Bruker D8 Discover X-ray diffractometer operating at $40 \mathrm{kV}$ and $30 \mathrm{~mA}$, employing Ni-filtered $\mathrm{Cu} \mathrm{K}_{\alpha 1}$ radiation $(\lambda=1.5406 \AA)$ and a position-sensitive LynxEye detector. A step size of $\Delta 2 \theta=0.05^{\circ}$ and a scan speed of $0.1 \mathrm{~s}$ per step were employed. 2D grazing-incident wide angle X-ray scattering (GIWAXS) data were collected using an Anton-Paar Saxspace system equipped with a $\mathrm{Cu} \mathrm{K}_{\alpha}$ microfocus source operated at $50 \mathrm{kV}$ and $1 \mathrm{~mA}$ and an Eiger Dectris R 1M 2D detector. SEM images were recorded with an FEI Helios Nanolab G3 UC DualBeam scanning electron microscope, operated at an acceleration voltage of $4 \mathrm{kV}$. AFM measurements were carried out with a NANOINK atomic force microscope in tapping mode with a scan rate of $0.3 \mathrm{~Hz}$, a proportional gain of 30 and an integral gain of 15 . UV-Vis absorption spectra were recorded using a Perkin Elmer Lambda 1050 spectrophotometer equipped with a $150 \mathrm{~mm}$ integrating sphere. Room temperature steady-state PL spectroscopy was performed with a Fluotime 300 spectrofluorometer (Picoquant). The samples were excited using a $405 \mathrm{~nm}$ (for $n=1$ or 2) laser or a $510 \mathrm{~nm}$ laser (for $n=3$ or $\mathrm{MAPbI}_{3}$ ) operated at $20 \mathrm{MHz}$ repetition rate and excited from the perovskite-coated side. To perform PL measurements as a function of temperature, the samples were placed in a closed cycle helium cryostat. The excitation source was a $485 \mathrm{~nm}$ pulsed laser (PicoQuant) with a repetition rate of $2 \mathrm{MHz}$ and an average power of $0.6 \mu \mathrm{W}$. A mechanical chopper was used to measure the quasi-PL signal. The emitted PL was dispersed by a spectrometer (Acton SP2500i, Princeton Instruments) and measured by a GaAs photomultiplier tube (Photonic Solutions). The quasi-PL signal was recorded by a lock-in amplifier (SR830, Stanford Research). Depth profiles of perovskite films on glass/FTO/ $/ \mathrm{TiO}_{2}$ substrates were measured with a ToF-SIMS 5 setup from IONTOF GmbH. Sputtering was performed using $\mathrm{Ar}^{+}$-clusters with $2.5 \mathrm{keV}$ ion energy on a 300 $\times 300 \mu \mathrm{m}^{2}$ raster size. Inside this sputter region an area of about $100 \times 100 \mu \mathrm{m}^{2}$ was analyzed using $\mathrm{Bi}_{3}{ }^{+}$ions with $30 \mathrm{keV}$ ion energy.

\section{Conflicts of interest}

There are no conflicts to declare. 
Electronic Supplementary Information (ESI): Additional experimental details, XRD and GIWAXS patterns, SEM images and temperature-dependent PL spectra.

\section{Acknowledgements}

The authors thank Dr. Steffen Schmidt from the LMU Munich for the SEM images. This project was financed by grants from the Federal Ministry of Education and Research (BMBF) under the project ID 03SF0516B and project ID 03SF0514A/B. The authors acknowledge funding from the Bavarian Collaborative Research Program "Solar Technologies Go Hybrid” (SolTech), the Center for NanoScience (CeNS), and the DFG Excellence Cluster "Nanosystems Initiative Munich" (NIM). D. A. acknowledges the financial support of the Engineering and Physical Science Research council (EPSRC) through the research grant EP/M025012/1. P. R. F. B. acknowledges funding through the EPSRC projects EP/M025020/1 and EP/M014797/1. P.D. also thanks the EPSRC for funding (EP/P03148X/1).

\section{ORCID}

Yinghong Hu: 0000-0002-9630-1662

Diego Alonso-Álvarez: 0000-0002-0060-9495

Piers R. F. Barnes: 0000-0002-7537-8759

Pablo Docampo: 0000-0001-6164-4748

\section{References}


1. H. Tsai, W. Nie, J.-C. Blancon, C. C. Stoumpos, R. Asadpour, B. Harutyunyan, A. J. Neukirch, R. Verduzco, J. J. Crochet, S. Tretiak, L. Pedesseau, J. Even, M. A. Alam, G. Gupta, J. Lou, P. M. Ajayan, M. J. Bedzyk, M. G. Kanatzidis and A. D. Mohite, Nature, 2016, 536, 312.

2. C. C. Stoumpos, D. H. Cao, D. J. Clark, J. Young, J. M. Rondinelli, J. I. Jang, J. T. Hupp and M. G. Kanatzidis, Chem. Mater., 2016, 28, 2852-2867.

3. S. A. Veldhuis, P. P. Boix, N. Yantara, M. Li, T. C. Sum, N. Mathews and S. G. Mhaisalkar, Adv. Mater., 2016, 28, 6804-6834.

4. N. Wang, L. Cheng, R. Ge, S. Zhang, Y. Miao, W. Zou, C. Yi, Y. Sun, Y. Cao, R. Yang, Y. Wei, Q. Guo, Y. Ke, M. Yu, Y. Jin, Y. Liu, Q. Ding, D. Di, L. Yang, G. Xing, H. Tian, C. Jin, F. Gao, R. H. Friend, J. Wang and W. Huang, Nat Photon, 2016, 10, 699-704.

5. E. R. Dohner, A. Jaffe, L. R. Bradshaw and H. I. Karunadasa, J. Am. Chem. Soc., 2014, 136, 13154-13157.

6. M. E. Kamminga, H.-H. Fang, M. R. Filip, F. Giustino, J. Baas, G. R. Blake, M. A. Loi and T. T. M. Palstra, Chem. Mater., 2016, 28, 4554-4562.

7. X. Zhang, X. Ren, B. Liu, R. Munir, X. Zhu, D. Yang, J. Li, Y. Liu, D.-M. Smilgies, R. Li, Z. Yang, T. Niu, X. Wang, A. Amassian, K. Zhao and S. Liu, Energy. Environ. Sci., 2017, DOI: $10.1039 / \mathrm{C} 7 \mathrm{EE} 01145 \mathrm{H}$.

8. R. Li, C. Yi, R. Ge, W. Zou, L. Cheng, N. Wang, J. Wang and W. Huang, Appl. Phys. Lett., 2016, 109, 151101.

9. S. Kumar, J. Jagielski, N. Kallikounis, Y. H. Kim, C. Wolf, F. Jenny, T. Tian, C. Hofer, Y.-C. Chiu, W. J. Stark, T.-W. Lee and C.-J. Shih, Nano Lett., 2017, DOI: 10.1021/acs.nanolett.7b01544.

10. J. Calabrese, N. L. Jones, R. L. Harlow, N. Herron, D. L. Thorn and Y. Wang, J. Am. Chem. Soc., 1991, 113, 2328-2330.

11. T. Ishihara, J. Takahashi and T. Goto, Solid State Commun., 1989, 69, 933-936.

12. D. B. Mitzi, J. Chem. Soc., Dalton Trans., 2001, DOI: 10.1039/B007070J, 1-12.

13. I. C. Smith, E. T. Hoke, D. Solis-Ibarra, M. D. McGehee and H. I. Karunadasa, Angew. Chem. Int. Ed., 2014, 53, 11232-11235.

14. D. H. Cao, C. C. Stoumpos, O. K. Farha, J. T. Hupp and M. G. Kanatzidis, J. Am. Chem. Soc., 2015, 137, 7843-7850.

15. Y. Hu, J. Schlipf, M. Wussler, M. L. Petrus, W. Jaegermann, T. Bein, P. MullerBuschbaum and P. Docampo, ACS Nano, 2016, 10, 5999-6007.

16. T. M. Koh, K. Thirumal, H. S. Soo and N. Mathews, ChemSusChem, 2016, 9, 2541-2558.

17. C. Ma, C. Leng, Y. Ji, X. Wei, K. Sun, L. Tang, J. Yang, W. Luo, C. Li, Y. Deng, S. Feng, J. Shen, S. Lu, C. Du and H. Shi, Nanoscale, 2016, 8, 18309-18314.

18. L. N. Quan, M. Yuan, R. Comin, O. Voznyy, E. M. Beauregard, S. Hoogland, A. Buin, A. R. Kirmani, K. Zhao, A. Amassian, D. H. Kim and E. H. Sargent, J. Am. Chem. Soc., 2016, 138, 2649-2655.

19. Z. Yuan, Y. Shu, Y. Xin and B. Ma, Chem. Commun., 2016, 52, 3887-3890.

20. R. L. Milot, R. J. Sutton, G. E. Eperon, A. A. Haghighirad, J. Martinez Hardigree, L. Miranda, H. J. Snaith, M. B. Johnston and L. M. Herz, Nano Lett., 2016, 16, 7001-7007.

21. W. Peng, J. Yin, K.-T. Ho, O. Ouellette, M. De Bastiani, B. Murali, O. El Tall, C. Shen, X. Miao, J. Pan, E. Alarousu, J.-H. He, B. S. Ooi, O. F. Mohammed, E. Sargent and O. M. Bakr, Nano Lett., 2017, 17, 4759-4767. 
22. X. Wu, M. T. Trinh, D. Niesner, H. Zhu, Z. Norman, J. S. Owen, O. Yaffe, B. J. Kudisch and X. Y. Zhu, J. Am. Chem. Soc., 2015, 137, 2089-2096.

23. Z. Guo, X. Wu, T. Zhu, X. Zhu and L. Huang, ACS Nano, 2016, 10, 9992-9998.

24. C. C. Stoumpos, C. M. M. Soe, H. Tsai, W. Nie, J.-C. Blancon, D. H. Cao, F. Liu, B. Traoré, C. Katan, J. Even, A. D. Mohite and M. G. Kanatzidis, Chem, 2017, 2, 427-440.

25. S. Zhang, P. Audebert, Y. Wei, A. Al Choueiry, G. Lanty, A. Bréhier, L. Galmiche, G. Clavier, C. Boissière, J.-S. Lauret and E. Deleporte, Materials, 2010, 3, 3385.

26. M. L. Petrus, Y. Hu, D. Moia, P. Calado, A. M. A. Leguy, P. R. F. Barnes and P. Docampo, ChemSusChem, 2016, 9, 2699-2707.

27. D. B. Mitzi, D. R. Medeiros and P. R. L. Malenfant, Inorg. Chem., 2002, 41, 2134-2145.

28. S. Ahmad, P. K. Kanaujia, H. J. Beeson, A. Abate, F. Deschler, D. Credgington, U. Steiner, G. V. Prakash and J. J. Baumberg, ACS Appl. Mater. Interfaces, 2015, 7, 25227-25236.

29. S. Gonzalez-Carrero, G. M. Espallargas, R. E. Galian and J. Perez-Prieto, J. Mater. Chem. A, 2015, 3, 14039-14045.

30. Y. Lin, Y. Bai, Y. Fang, Q. Wang, Y. Deng and J. Huang, ACS Energy Lett., 2017, DOI: 10.1021/acsenergylett.7b00442, 1571-1572.

31. Q. Shang, Y. Wang, Y. Zhong, Y. Mi, L. Qin, Y. Zhao, X. Qiu, X. Liu and Q. Zhang, J. Phys. Chem. Lett., 2017, 8, 4431-4438.

32. J. Liu, J. Leng, K. Wu, J. Zhang and S. Jin, J. Am. Chem. Soc., 2017, 139, 1432-1435.

33. J. A. Koza, J. C. Hill, A. C. Demster and J. A. Switzer, Chem. Mater., 2016, 28, 399-405.

34. B. J. Foley, J. Girard, B. A. Sorenson, A. Z. Chen, J. Scott Niezgoda, M. R. Alpert, A. F. Harper, D.-M. Smilgies, P. Clancy, W. A. Saidi and J. J. Choi, J. Mater. Chem. A, 2017, 5, 113-123.

35. J. Stevenson, B. Sorenson, V. H. Subramaniam, J. Raiford, P. P. Khlyabich, Y.-L. Loo and P. Clancy, Chem. Mater., 2017, 29, 2435-2444.

36. Y. Hu, J. Schlipf, M. Wussler, M. L. Petrus, W. Jaegermann, T. Bein, P. MüllerBuschbaum and P. Docampo, ACS Nano, 2016, 10, 5999-6007.

37. A. N. Jumabekov, E. Della Gaspera, Z. Q. Xu, A. S. R. Chesman, J. van Embden, S. A. Bonke, Q. Bao, D. Vak and U. Bach, J. Mater. Chem. C, 2016, 4, 3125-3130.

38. X. Lin, A. N. Jumabekov, N. N. Lal, A. R. Pascoe, D. E. Gómez, N. W. Duffy, A. S. R. Chesman, K. Sears, M. Fournier, Y. Zhang, Q. Bao, Y.-B. Cheng, L. Spiccia and U. Bach, Nat. Commun., 2017, 8, 613.

39. J. Liu, Y. Xue, Z. Wang, Z.-Q. Xu, C. Zheng, B. Weber, J. Song, Y. Wang, Y. Lu, Y. Zhang and Q. Bao, ACS Nano, 2016, 10, 3536-3542.

40. S. P. Senanayak, B. Yang, T. H. Thomas, N. Giesbrecht, W. Huang, E. Gann, B. Nair, K. Goedel, S. Guha, X. Moya, C. R. McNeill, P. Docampo, A. Sadhanala, R. H. Friend and H. Sirringhaus, Sci. $A d v ., 2017, \mathbf{3}$, e1601935. 